\title{
Aves do Parque Nacional da Serra do Cipó: o Vale do Rio Cipó, Minas Gerais, Brasil
}

\author{
Marcos Rodrigues, Lucas A. Carrara, Luciene P. Faria \& Henrique B. Gomes
}

Laboratório de Ornitologia, Departamento de Zoologia, ICB, Universidade Federal de Minas Gerais. Caixa Postal 486, 31270-901 Belo Horizonte, Minas Gerais, Brasil. E-mail: ornito@mono.icb.ufmg.br

\begin{abstract}
The birds of "Parque Nacional da Serra do Cipó": The Rio Cipó valley, Minas Gerais, Brazil. It is presented a bird survey of Rio Cipó valley from May 1998 to November 2002. The region is within the premises of 'Parque Nacional da Serra do Cipó', one of the most important conservation units of southeastern Brazil. Bird census were carried out through transect, mist-net captures and recording bird vocalizations. Species richness was estimated by jackknife method. It was recorded 226 species, belonging to 43 families. This represents $27 \%$ of all 837 bird species recorded at the Cerrado (a Savannah-like biome) region of central Brazil. A total of 2,249 bird individuals were captured with 4,486.82 net-hours. This represented 119 bird species of 23 families. Species richness was estimated at $239 \pm 5$ species. Six species are considered endemic to this biome: Augastes scutatus (Temminck, 1824) (Trochilidae), Hylocryptus rectirostris (Wied-NeuWied, 1821) (Furnariidae), Antilophia galeata (Lichtenstein, 1832) (Pipridae), Cyanocorax cristatellus (Temminck, 1823) (Corvidae), Charitospiza eucosma (Oberholser, 1905), Saltator atricollis (Vieillot, 1817), and Porphyrospiza caerulescens (Wied-Neuwied, 1830) (Emberizidae). Three species are considered nearthreatened to extinction: Sarcoramphus papa (Linnaeus, 1758) (Cathartidae), Cypsnagra hirundinacea (Lesson, 1831), and Charitospiza eucosma (Emberizidae). The Rio Cipó valley holds a significant number of the Cerrado bird species. Some habitats within this biome are becoming rare in the Cerrado region such as gallery forests, and the temporary lagoons along the rivers. Even waterfalls are disappearing in other regions of Brazil. In this sense, the "Parque Nacional da Serra do Cipó' is achieving one of its aims, which is biodiversity conservation.

KEY WORDS. Avian survey, biodiversity, Espinhaço Range, Cerrado, conservation units.
\end{abstract}

RESUMO. Foi conduzido um levantamento de espécies de aves do Vale do alto Rio Cipó durante o período de maio de 1998 a novembro de 2002. A região está totalmente inserida em uma das unidades de conservação mais importantes do sudeste do Brasil, o Parque Nacional da Serra do Cipó, em Minas Gerais. O método utilizado foi o de observação direta ao longo de 'transectos', captura com redes e identificação a partir do uso de vocalizações. A riqueza de espécies foi estimada usando-se o método de 'jackknife'. Foram registradas 226 espécies de aves pertencentes a 43 famílias. Isso corresponde cerca de $27 \%$ das 837 espécies já registradas para o bioma do Cerrado. Foram capturados 2.249 indivíduos num total de 4.486,82 horas-rede, onde foram amostradas 119 espécies pertencentes a 23 famílias. A riqueza foi estimada em $239 \pm 5$ espécies. Constam nesta lista seis espécies endêmicas do Cerrado: Augastes scutatus (Temminck, 1824) (Trochilidae), Hylocryptus rectirostris (Wied-NeuWied, 1821) (Furnariidae), Antilophia galeata (Lichtenstein, 1832) (Pipridae), Cyanocorax cristatellus (Temminck, 1823) (Corvidae), Charitospiza eucosma (Oberholser, 1905), Saltator atricollis (Vieillot, 1817), e Porphyrospiza caerulescens (Wied-Neuwied, 1830) (Emberizidae). Ocorrem também três espécies quase-ameaçadas de extinção: Sarcoramphus papa (Linnaeus, 1758) (Cathartidae), Cypsnagra hirundinacea (Lesson, 1831) e Charitospiza eucosma (Emberizidae). O Vale do Rio Cipó abriga uma porção significativa da avifauna do Cerrado. Alguns dos habitat encontrados no Vale estão se tornando cada vez mais raros na região do Cerrado de todo o Brasil, como as matas ciliares e o sistema de lagoas temporárias ao longo dos rios. Mesmo as cachoeiras, habitat importante para várias espécies, vêm desaparecendo em outras regiões do Brasil. Nesse sentido, a região do Vale do Rio Cipó dentro Parque consolida um dos seus objetivos que é a conservação da biodiversidade.

PALAVRAS CHAVE. Avifauna, biodiversidade, Cadeia do Espinhaço, Cerrado, unidades de conservação.

A criação de Unidades de Conservação em diversos estados brasileiros tem sido uma das poucas alternativas para a conservação in situ da biodiversidade (PRIMACK 1998). Entretanto, a maioria destas áreas não possui um levantamento dessa riqueza biológica (CAMARgos 2001). O inventariamento de espécies é o procedimento inicial e fundamental para que qualquer medida de manejo de uma Unidade de Conservação possa ser implementada (WILSON 1997).

Revista Brasileira de Zoologia 22 (2): 326-338, junho 2005 
O objetivo do presente trabalho foi o de fazer um levantamento das espécies de aves que ocorrem numa das Unidades de Conservação mais importantes do Brasil em termos de endemismo vegetal (Giullietr et al. 1997), endemismos de aves (Melo Júnior et al. 2001), e de divisor de águas de duas grandes bacias: o Parque Nacional da Serra do Cipó. Embora a Serra do Cipó tenha sido visitada por alguns naturalistas, desde os tempos do ciclo do ouro, poucos deles deixaram relatos públicos acessíveis (veja resumo histórico em PINTo 1952). Mais recentemente, Willis \& ONIKI (1991) percorreram a região de entorno do Parque durante quatro dias, onde observaram 165 espécies. MeLo JúnIor et al. (2001) levantaram a avifauna ao longo da rodovia que contorna o Parque, com 91 dias de observações entre 1994 e 2000 , e puderam registrar 273 espécies. O presente trabalho apresenta, pela primeira vez, uma lista comentada das espécies que ocorrem dentro do Parque, especificamente na região baixa, o Vale do Rio Cipó, onde o Cerrado é o ambiente mais representativo.

\section{MATERIAL E MÉTODOS}

\section{Área de estudo}

O Parque Nacional da Serra do Cipó está localizado na porção sul da Cadeia do Espinhaço, entre os paralelos $19^{\circ} \mathrm{e}$ $20^{\circ} \mathrm{S}$ e $43^{\circ}$ e $44^{\circ} \mathrm{W}$ ao longo da Serra do Cipó que engloba seis Municípios do estado de Minas Gerais: Jaboticatubas, Santana do Riacho, Morro do Pilar, Itambé do Mato Dentro, Itabira e Nova União.

A porção sul da Cadeia do Espinhaço atua como divisor de águas de três grandes bacias inseridas cada uma nos grandes biomas do sudeste do Brasil: a leste a bacia do Rio Doce, domínio da Mata Atlântica; a oeste o Rio São Francisco no Cerrado; ao norte, a bacia do Rio Jequitinhonha na Caatinga (Fig. 1). Trata-se de uma área cuja geologia, vegetação e fauna formam um ecossistema único no planeta, sendo um importante centro de endemismo e de biodiversidade ainda muito pouco explorado cientificamente (DAviEs et al. 1997). Os campos rupestres desta formação são tidos como o centro mundial de especiação das famílias vegetais Eriocaulaceae e Velloziaceae, como também de subdivisões de certas famílias como Rubiaceae e Melastomataceae (EiTEN 1992).

Com relação a avifauna, a Cadeia do Espinhaço também possui importantes endemismos, sendo que quatro das 30 espécies de aves consideradas endêmicas do Cerrado estão restritas aos campos rupestres desta formação (segundo SILVA \& BATES 2002), sendo elas: o beija-flor-de-gravata Augastes scutatus (Temminck, 1824), o joão-cipó Asthenes luizae Vielliard, 1990, o papa-moscas-de-costas-cinzentas Polystictus superciliaris (WiedNeuwied, 1831) e o canário-rabudo Embernagra longicauda Strikland, 1844 (mas veja VASCONCELos et al. 2003 que discutem a distribuição geográfica destas duas últimas espécies).

O Parque caracteriza-se por altitudes de 800 a $1400 \mathrm{~m}$, em uma serra onde na vertente leste destaca-se a vegetação de Mata Atlântica e na oeste, o Cerrado (Fig. 1). Possui na sua

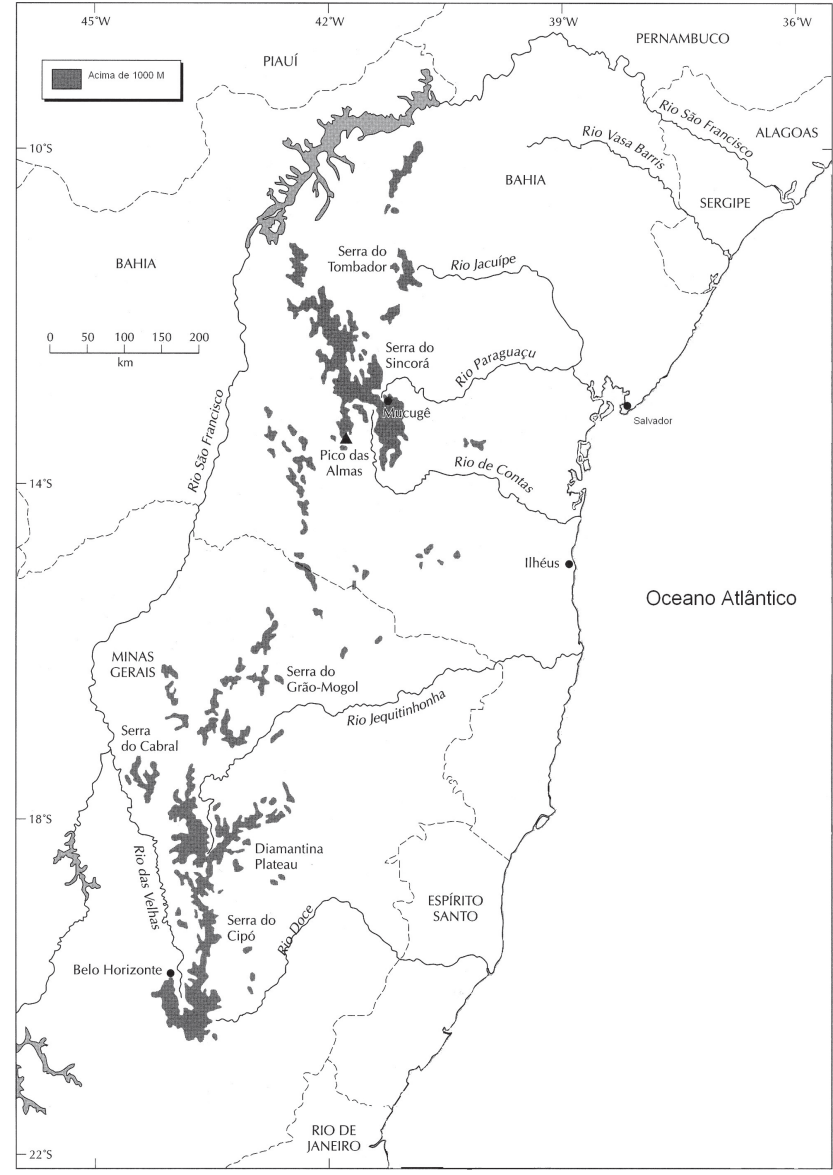

Figura 1. Localização da Cadeia do Espinhaço (áreas acima de 1000 $\mathrm{m})$, seus principais maciços nos estados de Minas Gerais e Bahia (leste do Brasil) e as bacias hidrográficas. O Parque Nacional da Serra do Cipó situa-se na serra homônima. Fonte: DAvies et al. 1997.

porção alta, como substrato rochoso mais comum, o quartzito, onde se desenvolve o campo rupestre (Ribeiro \& Walter 1998). $\mathrm{Na}$ sua região de baixada, vertente oeste, fica caracterizado o cerrado propriamente dito, apresentando áreas abertas (campos) e matas de galeria.

O clima da região é do tipo mesotérmico (Cwb de Köpen) com estações seca e chuvosa bem definidas e precipitação anual em torno de $1400 \mathrm{~mm}$. A temperatura da região tem pouca variação anual e mensal, mas a precipitação mostra-se com uma maior estacionalidade, com larga variação. Ocorrem quatro períodos estacionais bem definidos: a estação seca de maio a setembro; a estação chuvosa de novembro a janeiro; a estação pós-chuvosa de fevereiro a abril; e a estação pós-seca em outubro (MadeIRA \& Fernandes 1999).

As amostragens foram feitas de maio de 1998 a novembro de 2002 na região baixa do Parque, com altitude por volta de 800 m acima do nível do mar, ao longo do Vale do Rio Cipó, 
que se estende da entrada e sede administrativa do Parque

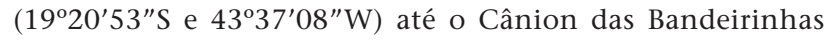
$\left(19^{\circ} 25^{\prime} 03^{\prime \prime}\right.$ e e $\left.43^{\circ} 34^{\prime} 16^{\prime \prime} \mathrm{W}\right)$. Trata-se de uma área situada na vertente oeste da Serra do Cipó com aproximadamente 1500 ha, onde são encontradas várias fisionomias do bioma cerrado segundo a classificação proposta por Ribeiro \& WALTER (1998): campo limpo, campo sujo, cerradão, mata ciliar, mata de galeria e mata semi-decídua. Além destes habitas, o Parque abriga várzeas com lagoas marginais, pastos em vários estágios de abandono, paredões rochosos e cachoeiras.

\section{Métodos}

As coletas de dados foram realizadas utilizando-se dois métodos distintos e complementares: (1) censo por observação direta (com e sem auxílio de 'play-back') e (2) captura com redes de neblina.

O censo por observação direta consiste em caminhar ao longo dos habitats específicos a partir do nascer do sol e anotar todas as espécies observadas ou ouvidas. Ao longo destas caminhadas foram feitas gravações das vocalizações não conhecidas. Estas foram feitas com um gravador cassete (Sony TCM 5000 EV) com microfone direcional (Sennheiser ME66). Em muitas ocasiões empregou-se a técnica do 'playback', que consiste em reproduzir o canto na direção da ave a fim de provocar a sua aproximação para fins de identificação.

A captura com 'redes de neblina' consiste da colocação de redes de 'nylon' (especialmente desenhadas para este tipo de atividade) de 12 , nove e seis $\mathrm{m}$ de comprimento por três $\mathrm{m}$ de altura. As aves capturadas foram identificadas ao nível de espécie, com auxílio de guias e chaves de identificação (Schauensee 1970, Grantsau 1988, Camargo 1986, Ridgely \& Tudor 1989, 1994, Sick 1997, Souza 2002) e marcados com anilhas metálicas numeradas fornecidas pelo CEMAVE (Centro de Pesquisas para Conservação das Aves Silvestres - IвAмA 1994). Foram feitas sessões de captura colocando-se de duas a 20 redes em todos os habitat encontrados no Vale.

Todos os indivíduos foram listados seguindo a nomenclatura e classificação de Sick (1997) com as modificações sugeridas pelo Свко (2003). O status de endemismo e conservação segue Silva (1995a), Collar et al. (1992), Machado et al. (1998) е Iвама (2003). Espécimes testemunhos foram depositados na Coleção Ornitológica do Departamento de Zoologia da Universidade Federal de Minas Gerais (licença IвAмA 207/2003 Fauna/MG).

Para cada espécie foi designada uma categoria de abundância segundo sua freqüência de ocorrência nos censos feitos em um habitat específico segundo NAKA et al. (2002): A (abundantes): espécies registradas entre $75 \%$ e $100 \%$ das visitas a área, isto é, com freqüência de ocorrência entre 75 e 100; C (comuns): com freqüência de ocorrência entre 50 e 74; E (escassas): com freqüência de ocorrência entre 25 e 49; R (raras): com freqüência de ocorrência entre 24 e 6; O (ocasionais): com freqüência menor que 5 .

As guildas alimentares a que as espécies pertencem são baseadas em Sick (1997) e observações ocasionais ao longo do estudo. Assim, as espécies foram classificadas como: O (onívoro), P (piscívoro), I (insetívoro), Ne (necrófago), Pl (planctívoro), C (carnívoro), F (frugívoro), G (granívoro) e N (nectarívoro). A denotação I/F, por exemplo, significa que a espécie é primariamente insetívora, mas se alimenta regularmente de frutos.

A riqueza de espécies foi estimada utilizando-se 'Jackknife de primeira-ordem' (Burnham \& Overton 1979, Heltshe \& ForRESTER 1983): $S_{j a c k 1}=S_{o b s}+Q_{1}\left(\frac{m-1}{m}\right)$ onde: $S_{j a c k 1}$ é o número de espécies estimado; $\mathrm{S}_{\mathrm{obs}}$ é o número total de espécies observado em todas as amostras; $\mathrm{Q}_{1}$ é número de espécies que ocorrem exatamente na amostra 1 e m é o número total de amostras. Os cálculos foram feitos utilizando-se o programa 'EstmateS' (Colwell 1997).

\section{RESULTADOS}

Foram registradas 226 espécies de aves pertencentes a 43 famílias. A maioria das espécies pertence a família Emberizidae (46 espécies) e Tyrannidae (45 espécies). A maioria das espécies que ocorrem na região foi amostrada (Fig. 2), sendo que a riqueza estimada foi de 239,71 $\pm 5,55$ espécies.

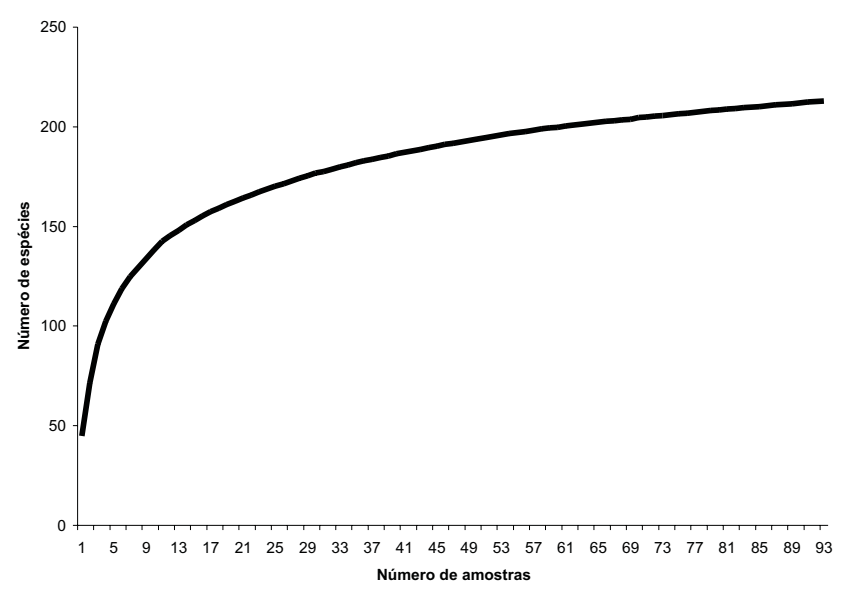

Figura 2. Acúmulo de espécies novas observadas ou capturadas nas amostragens feitas no Vale do Rio Cipó, Parque Nacional da Serra do Cipó durante o período de maio de 1998 a novembro de 2002.

Foram capturados 2.249 indivíduos num total de 4.486,82 horas-rede espalhadas por todos os meses do ano, onde foram amostradas 119 espécies pertencentes a 23 famílias. Isso corresponde a cerca de $55 \%$ do total de espécies que ocorrem no Vale.

Sessenta e nove espécies foram consideradas comuns, 59 escassas, 23 abundantes, 45 de ocorrência ocasional e 30 raras, segundo a freqüência de ocorrência. Foram reconhecidas 15 guildas alimentares, sendo 'insetívoros' (100 espécies), 'onívoros' (24) e 'granívoros' (20) as que apresentaram o maior nú-

Revista Brasileira de Zoologia 22 (2): 326-338, junho 2005 
mero de espécies (Anexo I).

Constam na lista seis espécies endêmicas do cerrado, uma espécie endêmica da Mata Atlântica, nenhuma espécie ameaçada de extinção no estado de Minas Gerais (segundo MACHADO et al. 1998) e nenhuma espécie ameaçada de extinção em nível nacional (segundo IBAma 2003). Três espécies são consideradas 'quase-ameaçadas' de extinção (segundo Collar et al. 1994): Sarcoramphus papa (Linnaeus, 1758) Cathartidae, Cypsnagra hirundinacea (Lesson, 1831) Emberizidae, e Charitospiza eucosma (Oberholser, 1905) Emberizidae (Anexo I).

\section{Registros importantes}

Ciconia maguari (Gmelin, 1789) (Maguari, Ciconiidae). É uma espécie de grande porte (140 cm de altura) de diagnose inconfundível. Três indivíduos foram observados freqüentando as lagoas temporárias da várzea do Rio Cipó em fevereiro de 1999. Os anos seguintes à observação destes três indivíduos foram mais secos do que o normal, o que parece ser uma tendência global (PARMESAN \& YoHE 2003). Sabe-se que as cegonhas não se reproduzem em anos muito secos (Kushlam 1986). Se a tendência climática de maior aridez for verdadeira, devido ao efeito estufa global, a espécie estaria sofrendo uma retração de sua área de distribuição geográfica.

Sarcoramphus papa (Linnaeus, 1758) (Urubu-rei, Cathartidae) - 'Quase-ameaçada'. É um visitante 'escasso' no Vale, onde foi observado apenas quatro vezes, geralmente sobrevoando o Cânion das Bandeirinhas. O maior grupo observado foi de seis indivíduos, pousados na mata de galeria do Rio Mascates após um longo período de chuvas. É uma espécie considerada 'quase-ameaçada' de extinção (Collar et al. 1992). Provavelmente, um indivíduo desta espécie necessita de uma área de vida grande (Schoener 1968). Devido à sua especificidade de dieta (grandes carcaças) e de nidificação (paredões rochosos), à competição com o urubu comum Coragyps atratus (Bechstein, 1793), cada vez mais abundante no entorno do Parque devido à intensa urbanização dos últimos cinco anos, é possível que suas populações dentro do mesmo estejam ameaçadas. Fazemse necessários censos periódicos e procura pelos sítios de nidificação a fim de que se possa estimar sua população e a viabilidade da mesma dentro do Parque.

Cypseloides senex (Temminck, 1826) (Andorinhão-velhoda-cascata, Apodidae). Trata-se de uma espécie que depende das grandes cachoeiras porque nidifica entre as cavidades formadas atrás da coluna d'água destas (SICK 1997). Este andorinhão foi observado nidificando nas cachoeiras da Farofa e da Taioba, ambas dentro dos limites do Parque. É uma espécie relativamente rara, que ocorre em bandos durante os meses chuvosos (outubro a fevereiro), mas pouco se sabe sobre o número de indivíduos ou de ninhos da população do Parque. Bandos com mais de 200 indivíduos já foram observados na cachoeira da Farofa nos mês de outubro. Não se sabe qual o destino destes indivíduos na estação seca.

Augastes scutatus (Temminck, 1824) (Beija-flor-de-gravata, Trochilidae) - 'Endêmico'. É um beija-flor endêmico da por- ção central e sul da serra do espinhaço e muito comum nas regiões acima de $1000 \mathrm{~m}$ dentro do Parque. É uma espécie ocasional no Vale, onde foi capturada apenas uma vez na Lagoa Comprida em 25/IX/1999, a menos de 800 m de altitude.

Malacoptila striata (Spix, 1824) (João-barbudo, Bucconidae) -'Endêmica'. Malacoptila striata foi observada em uma única oportunidade em área de mata ciliar próxima ao encontro dos Rios Cipó e Mascate. Silva (1995a) a classifica como espécie que ocorre no cerrado, enquanto Sick (1997) a coloca como endêmica da Mata Atlântica. A consideração de SıLVA (1995a) é baseada no trabalho de J. Reinhardt de 1870, que a coletou numa área de transição entre Mata Atlântica e Cerrado, no município de Lagoa Santa, Minas Gerais (PinTo 1952). Melo JúnIor et al. (2001) também registraram esta espécie na vertente leste (Mata Atlântica) e oeste (Cerrado) da Serra do Cipó. Este, e o presente registro mostram que a espécie ocorre em área de transição de Cerrado e Mata Atlântica.

Hylocryptus rectirostris (Wied-Neuwied, 1831) (Fura-barreira, Furnariidae) - 'Endêmica'. Esse passeriforme habita somente a porção densamente florestada das margens do Rio Cipó e Rio Mascates, onde foram observados alguns ninhos. É uma espécie endêmica das matas ciliares da região centro-sul do Brasil (Mato Grosso, sul de Goiás, extremo sul da Bahia, sul-sudoeste de Minas Gerais, oeste de São Paulo e noroeste do Paraná), chegando ao extremo leste do Paraguai (Del Hoyo et al. 2003). Hylocryptus rectirostris é uma espécie sensível a distúrbios ambientais e aparece como prioritária para pesquisa segundo STOTZ et al. (1996).

Antilophia galeata (Lichtenstein, 1832) (Soldadinho, Pipridae) - 'Endêmico'. É um passeriforme endêmico do Cerrado (SILva 1995a) e que habita o sub-bosque das matas ciliares que se formam ao longo dos rios do Vale. Sua categoria de freqüência de ocorrência é 'comum' (Anexo I).

Charitospiza eucosma (Oberholser, 1905) (Mineirinho, Emberizidae) - 'Endêmico', 'Quase-ameaçado'. Espécie endêmica do Cerrado (SILVA 1995a) de ocorrência 'ocasional' no Vale e rara em outras regiões do Cerrado. Um único indivíduo foi observado por alguns dias após uma grande queimada numa região próxima à sede do Parque. É uma espécie 'quaseameaçada' segundo Collar et al. (1992).

\section{Registros importantes no entorno da área do Parque}

Há duas espécies de interesse conservacionista (ameaçadas de extinção no estado de Minas Gerais segundo Machado et al. 1998) que já foram registradas no entorno do Parque e merecem ser destacadas.

Ara ararauna (Linnaeus, 1758) (Arara-de-barriga-amarela ou Canindé, Psittacidae). Há registros desta espécie sobrevoando o Km 74 da rodovia MG 010 durante a estação seca de 2002, e em Lagoa Santa (junho de 1997, julho de 1998 e agosto de 1999). Tais localidades distam a 15 e $40 \mathrm{~km}$ do Parque, respectivamente (Rodrigues \& Goulart 2004). A Canindé é uma espécie cujas populações vêm sofrendo declínio em todo Brasil central (SICK 1997) e hoje, no estado de Minas Gerais, é conhecida ape-

Revista Brasileira de Zoologia 22 (2): 326-338, junho 2005 
nas no extremo noroeste do estado (MACHADo et al. 1998).

Amazona aestiva (Linnaeus, 1758) (Papagaio-verdadeiro, Psittacidae). Registrada no entorno do Parque por Melo JúnIoR et al. (2001). Entretanto, estes autores não indicam a localidade e tão pouco sua freqüência de ocorrência. Está é uma espécie ainda relativamente comum na Área de Proteção Ambiental do Carste de Lagoa Santa, a $40 \mathrm{~km}$ do Parque, cuja população está ameaçada por caçadores que abastecem o mercado de animais de estimação (M. Rodrigues dados não publicados).

\section{DISCUSSÃO}

\section{Riqueza de espécies}

O vale do Rio Cipó está situado na vertente oeste da Serra do Cipó, que está inserido no bioma do Cerrado. O número de espécies que ocorrem no Vale corresponde à cerca de $26 \%$ das 837 espécies já registradas para o bioma do Cerrado (SiLVA 1995a). A composição de espécies é típica do Cerrado, com seus endemismos e Tyrannidae e Emberizidae como famílias dominantes em número de espécies (SiLva 1995b).

Existem apenas dois levantamentos de aves publicados que foram feitos na região da Serra do Cipó, sendo o primeiro com apenas quatro dias de observações em áreas no entorno do Parque (Willis \& ONIKI 1991). Estes autores registraram um total de 165 espécies para ambas as áreas, as baixas (correspondente à região do Rio Cipó, mas fora do Parque, com 87 espécies) e as altas (correspondentes a Conceição do Mato na vertente leste, Alto do Palácio e Chapéu do Sol à oeste, com 131 espécies). O segundo levantamento, feito com 91 dias de observações ao longo da rodovia que contorna o parque, registrou 248 espécies nas áreas altas e baixas da vertente oeste da serra (Melo JúNIOR et al. 2001). Destas, 76 espécies foram registradas nas áreas baixas. O presente trabalho registrou, apenas para a região baixa do vale do Rio Cipó uma riqueza muito maior do que previamente conhecida. Além disso, a riqueza estimada para área mostra que possivelmente cerca de outras 20 espécies podem ocorrer na área de estudo.

Além do elevado número de espécies em relação aos levantamentos anteriores, algumas delas não constam nas listas de Willis \& ONIKI e de Melo JúnIor et al., tais como: Pilherodius pileatus (Boddaert, 1783) e Tigrisoma lineatum (Boddaert, 1783) (Ardeidae), Ciconia maguari (Ciconiidae), Pardirallus maculatus (Boddaert, 1783) (Rallidae), Columbina minuta (Linnaeus, 1766) (Columbidae), Cypseloides senex (Apodidae), Anthracothorax nigricollis (Vieillott, 1817), Thalurania furcata (Gmelin, 1788), T. glaucopis (Gmelin, 1788), Heliomaster squamosus (Temminck, 1823) e Callyphlox amethystina (Boddaert, 1783) (Trochilidae), Phacellodomus ruber (Vieillot, 1819) (Furnariidae), Myiarchus swainsoni Cabanis \& Heine 1859 (Tyrannidae), Progne tapera (Linnaeus, 1766) (Hirundinidae), Thlypopsis sordida (d'Orbigny \& Lafiesnaye 1837), Thraupis palmarum (Wied, 1821), Sporophila plumbea (Wied, 1830), S. collaris (Boddaert, 1783), S. lineola (Linnaeus, 1758), S. leucoptera (Vieillot, 1817), Arremon taciturnus (Hermann,1783) (Emberizidae). Infelizmente, os levantamentos anteriores não mostraram o esforço de coleta empregado, o que dificulta comparações. A curva do coletor, como mostrada neste estudo, é uma ferramenta indispensável para que se possa saber o número de espécies real (pelo menos aproximado) que ocorre numa determinada área. A riqueza de espécies é geralmente confundida com 'número de espécies amostrado' na maioria dos levantamentos de aves feitos no Brasil. Métodos de rarefação, que estimam riqueza de espécies, permitem que comparações entre áreas e/ou diferentes levantamentos na mesma área, tenham sentido biológico e estatístico (veja Gotell \& Colwell 2001). Possivelmente, naqueles estudos anteriores, o esforço amostral não foi suficiente para se detectar muitas destas espécies.

\section{Conservação e manejo}

O Vale do Rio Cipó abriga uma porção significativa da avifauna do Cerrado, incluindo espécies endêmicas e ameaçadas de extinção. Além das espécies, alguns dos habitat encontrados no Vale estão se tornando cada vez mais raros na região do Cerrado de todo o Brasil, como as matas ciliares e o sistema de lagoas temporárias ao longo dos rios (WwF 1995). Mesmo as cachoeiras, habitat importante para várias espécies de aves vêm desaparecendo em outras regiões do Brasil, dando lugar às hidroelétricas, cada vez mais necessárias para o abastecimento de energia para a sempre crescente população humana. Nesse sentido, a região do Vale do Rio Cipó, dentro do Parque, consolida um dos seus objetivos que é a conservação da biodiversidade a partir da conservação destes habitats.

Por outro lado, o Vale sofre ameaças que comprometem este objetivo. Entre elas, destaca-se a erosão causada pela estrada que liga a sede do Parque à cachoeira da Farofa. Está é uma grande fonte de assoreamento dos rios e lagoas temporárias que formam o Vale. Um dos efeitos de médio e longo prazo seria a perda dos brejos e o empobrecimento da mata ciliar (o que já vem acontecendo), dois ambientes extremamente importantes do ponto de vista da avifauna.

Uma outra ameaça é a alta freqüência de incêndios que ocorre no final da estação seca. Nessa época (setembro e outubro) se dá o início do período reprodutivo de várias espécies, e portanto, o fogo deve ter um efeito negativo na dinâmica da população das mesmas.

Questões sobre a viabilidade em longo prazo de algumas populações de aves e ações de manejo que contemplem os objetivos de um Parque Nacional só poderão ser respondidas com estudos mais detalhados das mesmas. A presente lista de espécies é de fundamental importância para futuros planos de manejo dentro do Parque (Rodrigues et al. 2000).

\section{AGRADECIMENTOS}

Este trabalho não poderia ter sido realizado sem o apoio fundamental da "Fundação o Boticário de Proteção à Natureza". Somos gratos ainda à Pro-Reitoria de Pesquisa da Universidade Federal de Minas Gerais; ao Conselho Nacional para o Desenvolvimento Científico e Tecnológico (CNPq), pela concessão de uma bolsa de produtividade em pesquisa a M. Rodrigues e uma 
bolsa de iniciação científica a L. A. Carrara; ao IBAMA do PARNA Serra do Cipó. Agradecemos especialmente a T. Guerra pelo abrigo durante várias campanhas; L. Atzeni e D. Safar pelo apoio no campo; M.F. Vasconcelos pela ajuda na identificação, taxidermia, leitura do manuscrito e curadoria da Coleção Ornitológica do Departamento de Zoologia da UFMG; ao Centro de Pesquisa para Conservação das Aves Silvestres (CEMAVE-IBAMA) pela concessão das anilhas e licença para anilhamento de aves silvestres, a FUNDEP-UFMG pela administração financeira; ao Departamento de Zoologia da UFMG pelo apoio e infra-estrutura.

\section{REFERÊNCIAS BIBLIOGRÁFICAS}

Burnham, K.P. \& W.S. OverTon. 1979. Robust estimation of population size when capture probabilities vary among animals. Ecology, Davis, 60 (2): 927-936.

Camargo, H.F.A. 1986. Contribuição ao estudo das espécies brasileiras do gênero Elaenia (Aves, Tyrannidae). Boletim do Centro de Estudos Ornitológicos, São Paulo, 2 (1): 1-38.

Camargos, R.M.F. 2001. Unidades de Conservação em Minas Gerais: Levantamento e Discussão. Publicações Avulsas da Fundação Biodiversitas, Belo Horizonte, 2: 7-67.

Collar, N.J.; L.P. Gonzaga; N. Krabbe; A. Madroño Nieto; L.G. NARANJo; T.A. Parker III \& D.C. Wege. 1992. Threatened Birds of the Americas. Washington, Smithsonian Institution Press, 1150p.

Collar, N. J.; M. J. Crosby \& A.J. Stattersfield. 1994. Birds to watch 2: the world list of threatened birds. Cambridge, BirdLife International, Conservation Series 4, 407p.

Colwell, R. K. 1997. EstimateS: Statistical estimation of species richness and shared species from samples. Version 5, User's Guide and application published at: http://viceroy. eeb.uconn.edu/estimates.

Comitê Brasileiro de Registros Ornitológicos. 2003. Lista de aves do Brasil. Disponível na World Wide Web: http://www.ib. usp.br/cbro. Acesso em 20/II/2004.

Davies, S. D.; V. H. Heywood; O. Herrera-MacBride; J. Villa-Lobos \& A.C. Hamilton. 1997. Centres of Plant Diversity: A Guide and Strategy for their Conservation. The Americas. Cambridge, IUCN Publications Unit, vol. 3, 578p.

Del Hoyo, J.; A. Elliott \& D.A. Christie. 2003. Handbook of the birds of the world. Broadbills to Tapaculos. Barcelona, Lynx Edicions, vol. 8, 845p.

Erten, G. 1992. Natural Brazilian vegetation types and their causes. Anais da Academia Brasileira de Ciências, Rio de Janeiro, 64 (1): 34-65.

Giullieti, A.M.; J.R. Pirani \& R.M. Harley. 1997. Espinhaço Range region, Eastern Brazil, p. 397-404. In: S. D. DAVIES; V.H. Heywood; O. Herrera-MacBryde; J. Villa-Lobos \& A.C. HamilTON (Eds). Centres of plant diversity: a guide and strategy for their conservation. Oxford, Information Press, 578p.

Gotelli, N.J. \& R.K. Colwell. 2001. Quantifying biodiversity: procedures and pitfalls in the measurement and comparison of species richness. Ecology Letters, Montpellier, France, 4
(1): 379-391.

Grantsau, R. 1988. Die Kolibris Brasiliens. Rio de Janeiro, Expressão e Cultura, 233p.

HeLtSHE, J. \& N.E. ForRester. 1983. Estimating species richness using the jackknife procedure. Biometrics, Arlington, 39: 1-11.

Iвама. 1994. Manual de Anilhamento de Aves Silvestres. Brasília, Instituto Brasileiro do Meio Ambiente e dos Recursos Naturais Renováveis, $146 \mathrm{p}$.

Iвама. 2003. Lista das espécies de fauna ameaçada de extinção. Disponível na World Wide Web: http://www. ibama.gov.br/fauna/downloads/lista\%20spp.pdf. Acesso em 31/VII/2004.

Kushlam, J.A. 1986. Responses of wading birds to seasonally fluctuating water levels: strategies and their limits. Colonial Waterbirds, Lawrence, 9 (1): 155-162.

Machado, A.B.M.; G.A.B. Fonseca; R.B. Machado; L.M.S. Aguiar \& L.V. Lins. 1998. Livro vermelho das espécies ameaçadas de extinção da fauna de Minas Gerais. Belo Horizonte, Fundação Biodiversitas, 605p.

Madeira, A.J. \& W.G. Fernandes. 1999. Reprodutive phenology of sympatric taxa of Chamaecrista (Leguminosae) in Serra do Cipó, Brasil. Jornal of Tropical Ecology, Cambridge 15 (2): 463-479.

Melo Júnior, T.A.; M.F. Vasconcelos; G.W. Fernandes \& M.A. MARINI. 2001. Bird species distribution and conservation in Serra do Cipó, Minas Gerais, Brazil. Bird Conservation International, Cambridge, 11 (1): 189-204.

NaKA, L.N.; M. Rodrigues; A.L. Roos \& M.A.. Azevedo. 2002. Bird conservation on the island of Santa Catarina. Bird Conservation International, Cambridge, 12 (1): 123-150.

Parmesan, C. \& G. Yohe. 2003. A globally coherent fingerprint of climate change impacts across natural systems. Nature, London, 421: 37-42.

Pinto, O.M.O. 1952. Súmula histórica e sistemática da ornitologia de Minas Gerais. Arquivos de Zoologia, São Paulo, 8 (1): $1-51$.

Primack, R.B. 1998. Essentials of conservation biology. Sinderland, Sinauer, 564p.

Ribeiro, J.F. \& B.M.T. Walter. 1998. Fitofisionomias do bioma cerrado, p. 89-166. In: S.M. SANo \& S.P. AlmeIDA (Eds). Cerrado: Ambiente e Flora. Planaltina, Embrapa, 556p.

Ridgely, R.S. \& G. Tudor. 1989. The birds of South America. Oxford, Oxford University Press, vol. 1, 516p.

Ridgely, R.S. \& G. Tudor. 1994. The birds of South America. Oxford, Oxford University Press, vol. 2, 81p.

Rodrigues, M.; L. Carrara \& L.P. Faria. 2000. Avifauna como ferramenta para o monitoramento de Unidades de Conservação. In: Anais do II Congresso Brasileiro de Unidades de Conservação, Campo Grande, Universidade Livre do Meio Ambiente, vol. 2, p. 356-364.

Rodrigues, M. \& F.F. Goulart. 2004. Aves Regionais: de Burton aos dias de hoje, p. 590-603. In: A.H. Lisboa \& E.M.A. GoularT (Eds). Navegando o Rio das Velhas das Minas aos Gerais. 
Belo Horizonte, Instituto Guaicuy-SOS Rio das Velhas, Projeto Manuelzão, UFMG, 755p.

Schauensee, R.M. 1970. A guide to the birds of South America. Philadelphia, John Wiley and Sons, 498p.

Schoener, T.W. 1968. Sizes of feeding territories among birds. Ecology, Davis, Califórnia, 49 (1): 123-141.

SICK, H. 1997. Ornitologia brasileira. Rio de Janeiro, Nova fronteira, $912 \mathrm{p}$.

SiLva, J.M.C. 1995a. Birds of the Cerrado Region, South America. Steenstrupia, Copenhagen, 21 (2): 69-92.

SiLVA, J.M.C. 1995b. Biogeographic analysis of the South American Cerrado avifauna. Steenstrupia, Copenhagen, 21 (1): 49-67.

Silva, J.M.C. \& J. M. Bates. 2002. Biogeographic patterns and conservation in the South American Cerrado: A tropical savanna hostspot. BioScience, Washington, 52 (3): 225-233.

SouzA, D. 2002. All the birds of Brazil: An identification guide.

Dall, Feira de Santana, 356p.
Stotz, D.F.; J.W. Fitzpatrick; T.A. Parker III \& D.K. Moskovits. 1996. Neotropical Birds: ecology and conservation. Chicago, The University of Chicago Press, 478p.

Vasconcelos, M.F.; M. Maldonado-Coelho \& D.R.C. Buzzetti. 2003. Range extensions for the Grey-backed Tachuri (Polystictus superciliaris) and the Pale-throated Serra-finch (Embernagra longicauda) with a revision of their geographic distribution. Ornitología Neotropical, St louis, 14 (4): 477-489.

Willis, E.O. \& Y. ONIKI. 1991. Avifaunal transects across the open zones of northern Minas Gerais, Brazil. Ararajuba, Rio de Janeiro, 2 (1): 41-58.

WiLson, E.O. 1997. A situação atual da diversidade biológica, p. 3-24. In: E.O. WiLson (Ed.) Biodiversidade. Rio de Janeiro, Editora Nova Fronteira, 657p.

WwF (Fundo Mundial para a Natureza). 1995. De grão em grão, o cerrado perde o espaço - Cerrado: impactos do processo de ocupação. Documentos para discussão. Brasília, WWF, 66p.

Anexo I. Lista de espécies de aves registradas no Vale do Rio Cipó, região de cerrado do Parque Nacional da Serra do Cipó, em Minas Gerais (*espécimes testemunhos depositados na Coleção Ornitológica do Departamento de Zoologia da Universidade Federal de Minas Gerais). Classificação taxonômica e nomes populares segundo SıCK (1997) com as modificações sugeridas pelo CBRO (2003). Conservação e Endemismo: (ENC) espécies endêmicas do Cerrado segundo Sılva (1995); (EMA) espécies endêmicas da Mata Atlântica (segundo Sıck 1997); (NT) espécies 'quase-ameaçadas' (segundo Collar et al. 1992). Abundância relativa: (A) abundantes: espécies registradas entre $75 \%$ e $100 \%$ das visitas a área, isto é, com freqüência de ocorrência entre 75 e 100; (C) comuns: com freqüência de ocorrência entre 50 e 74; (E) escassas: com freqüência de ocorrência entre 25 e 49; $(\mathrm{R})$ raras: com freqüência de ocorrência entre 24 e 6; (O) ocasionais: com freqüência menor que 5. Guilda alimentar: $(\mathrm{O})$ onívoro, $(\mathrm{P})$ piscívoro, (I) insetívoro, $(\mathrm{Ne})$ necrófago, (PI) planctívoro, (C) carnívoro, (F) frugívoro, $(\mathrm{G})$ granívoro, $(\mathrm{N})$ nectarívoro, $(\mathrm{Cn})$ carcinófago.

\begin{tabular}{|c|c|c|c|c|}
\hline Famílias e espécies & Nome popular & $\begin{array}{c}\text { Conservação e } \\
\text { endemismo }\end{array}$ & $\begin{array}{c}\text { Abundância } \\
\text { relativa }\end{array}$ & $\begin{array}{c}\text { Guilda } \\
\text { alimentar }\end{array}$ \\
\hline \multicolumn{5}{|l|}{ Tinamidae } \\
\hline Crypturellus parvirostris (Wagler, 1827) & Inhambu-chororó & & $\mathrm{C}$ & $\mathrm{O}$ \\
\hline Crypturellus tataupa (Temminck, 1815) & Inhambu-chintã & & $\mathrm{R}$ & $\mathrm{O}$ \\
\hline Rhynchotus rufescens (Temminck, 1815) & Perdiz & & $\mathrm{C}$ & $\mathrm{O}$ \\
\hline Nothura maculosa (Temminck, 1815) & Codorna-amarela & & $\mathrm{E}$ & $\mathrm{O}$ \\
\hline \multicolumn{5}{|l|}{ Podicipedidae } \\
\hline Tachybaptus dominicus (Linnaeus, 1766) & Mergulhão-pequeno & & $\mathrm{O}$ & $\mathrm{P}$ \\
\hline \multicolumn{5}{|l|}{ Phalacrocoracidae } \\
\hline Phalacrocorax brasilianus (Gmelin, 1789) & Biguá & & $\mathrm{O}$ & $P$ \\
\hline \multicolumn{5}{|l|}{ Ardeidae } \\
\hline Ardea cocoi Linnaeus, 1766 & Garça-moura & & $\mathrm{O}$ & $P$ \\
\hline Ardea alba Linnaeus, 1758 & Garça-branca-grande & & $\mathrm{R}$ & $P$ \\
\hline Egretta thula (Molina, 1782) & Garça-branca-pequena & & $\mathrm{R}$ & $P$ \\
\hline Butorides striatus (Linnaeus, 1758) & Socozinho & & $\mathrm{E}$ & $\mathrm{P} / \mathrm{I}$ \\
\hline Syrigma sibilatrix (Temminck, 1824) & Maria-faceira & & $\mathrm{C}$ & 1 \\
\hline Pilherodius pileatus (Boddaert, 1783) & Garça-real & & $\mathrm{E}$ & I/P \\
\hline Nycticorax nycticorax (Linnaeus, 1758) & Savacu & & $\mathrm{O}$ & $P$ \\
\hline Tigrisoma lineatum (Boddaert, 1783) & Socó-boi-ferrugem & & $\mathrm{O}$ & $P$ \\
\hline \multicolumn{5}{|l|}{ Ciconiidae } \\
\hline Ciconia maguari (Gmelin, 1789) & Maguari & & $\mathrm{O}$ & $\mathrm{O}$ \\
\hline
\end{tabular}


Anexo I. Continuação.

\begin{tabular}{|c|c|c|c|c|}
\hline Famílias e espécies & Nome popular & $\begin{array}{c}\text { Conservação e } \\
\text { endemismo }\end{array}$ & $\begin{array}{c}\text { Abundância } \\
\text { relativa }\end{array}$ & $\begin{array}{c}\text { Guilda } \\
\text { alimentar }\end{array}$ \\
\hline \multicolumn{5}{|l|}{ Cathartidae } \\
\hline Sarcoramphus papa (Linnaeus, 1758) & Urubu-rei & NT & $\mathrm{E}$ & $\mathrm{Ne}$ \\
\hline Coragyps atratus (Bechstein, 1793) & Urubu-de-cabeça-preta & & $\mathrm{C}$ & $\mathrm{Ne}$ \\
\hline Cathartes aura (Linnaeus, 1758) & Urubu-de-cabeça-vermelha & & A & $\mathrm{Ne}$ \\
\hline \multicolumn{5}{|l|}{ Anatidae } \\
\hline Dendrocygna viduata (Linnaeus, 1766) & Irerê & & $\mathrm{E}$ & $\mathrm{PI}$ \\
\hline Amazonetta brasiliensis (Gmelin, 1789) & Marreca-pé-vermelho & & $\mathrm{C}$ & $\mathrm{PI}$ \\
\hline \multicolumn{5}{|l|}{ Accipitridae } \\
\hline Elanus leucurus (Vieillot, 1818) & Peneira & & $\mathrm{O}$ & $\mathrm{C}$ \\
\hline Elanoides forficatus (Linnaeus, 1758) & Gavião-tesoura & & $\mathrm{O}$ & $\mathrm{C}$ \\
\hline Accipter bicolor (Vieillot, 1817) & Gavião-bombachinha-grande & & $\mathrm{O}$ & $\mathrm{C} / \mathrm{I}$ \\
\hline Geranoaetus melanoleucus (Vieillot, 1819) & Águia-chilena & & $\mathrm{R}$ & $\mathrm{C}$ \\
\hline Buteo albicaudatus Vieillot, 1816 & Gavião-de-cauda-branca & & $\mathrm{R}$ & $\mathrm{C}$ \\
\hline Rupornis magnirostris (Gmelin, 1789) & Gavião-carijó & & $\mathrm{C}$ & $\mathrm{C} / \mathrm{I}$ \\
\hline Heterospizias meridionalis Sharpe, 1874 & Gavião-caboclo & & $\mathrm{E}$ & $\mathrm{C}$ \\
\hline Geranospiza caerulescens (Vieillot, 1817) & Gavião-pernilongo & & $\mathrm{O}$ & $\mathrm{C}$ \\
\hline \multicolumn{5}{|l|}{ Falconidae } \\
\hline Herpetotheres cachinnans (Linnaeus, 1758) & Acauã & & $\mathrm{C}$ & $\mathrm{C} / \mathrm{I}$ \\
\hline Micrastur semitorquatus (Vieillot, 1817) & Gavião-relógio & & $\mathrm{O}$ & $\mathrm{C} / \mathrm{I}$ \\
\hline Milvago chimachima (Vieillot, 1816) & Carrapateiro & & $\mathrm{C}$ & $\mathrm{O}$ \\
\hline Caracara plancus (Miller, 1777) & Carcará & & $\mathrm{C}$ & $\mathrm{O}$ \\
\hline Falco femoralis Temminck, 1822 & Falcão-de-coleira & & $\mathrm{E}$ & $\mathrm{C}$ \\
\hline Falco sparverius Linnaeus, 1758 & Quiriquiri & & $\mathrm{E}$ & $\mathrm{C} / \mathrm{I}$ \\
\hline \multicolumn{5}{|l|}{ Cracidae } \\
\hline Penelope superciliaris Temmink, 1815 & Jacu & & $\mathrm{O}$ & $\mathrm{F}$ \\
\hline \multicolumn{5}{|l|}{ Aramidae } \\
\hline Aramus guarauna (Linnaeus, 1766) & Carão & & $\mathrm{O}$ & $\mathrm{Cn}$ \\
\hline \multicolumn{5}{|l|}{ Rallidae } \\
\hline Pardirallus maculatus Boddaert, 1783 & Saracura-carijó & & $\mathrm{O}$ & $\mathrm{O}$ \\
\hline Aramides cajanea (Statius Muller, 1766) & Saracura-três-potes & & $\mathrm{R}$ & $\mathrm{O}$ \\
\hline Porzana albicollis (Vieillot, 1819) & Sanã-carijó & & $\mathrm{E}$ & $\mathrm{O}$ \\
\hline Porphyrio martinica (Linnaeus, 1766) & Frango-d'água-azul & & $\mathrm{O}$ & $\mathrm{O}$ \\
\hline \multicolumn{5}{|l|}{ Cariamidae } \\
\hline Cariama cristata (Linnaeus, 1766) & Seriema & & $\mathrm{C}$ & $\mathrm{O}$ \\
\hline \multicolumn{5}{|l|}{ Jacanidae } \\
\hline Jacana jacana (Linnaeus, 1766) & Jaçanã & & $\mathrm{C}$ & $\mathrm{O}$ \\
\hline \multicolumn{5}{|l|}{ Charadriidae } \\
\hline Vanellus chilensis (Molina, 1782) & Quero-quero & & $\mathrm{C}$ & 1 \\
\hline \multicolumn{5}{|l|}{ Scolopacidae } \\
\hline Tringa flavipes (Gmelin, 1789) & Maçarico-de-perna-amarela & & $\mathrm{O}$ & $\mathrm{O}$ \\
\hline Gallinago paraguaiae (Vieillot, 1816) & Narceja & & $\mathrm{E}$ & $\mathrm{O}$ \\
\hline
\end{tabular}


Anexo I. Continuação.

\begin{tabular}{|c|c|c|c|c|}
\hline Famílias e espécies & Nome popular & $\begin{array}{c}\text { Conservação e } \\
\text { endemismo }\end{array}$ & $\begin{array}{l}\text { Abundância } \\
\text { relativa }\end{array}$ & $\begin{array}{l}\text { Guilda } \\
\text { alimentar }\end{array}$ \\
\hline \multicolumn{5}{|l|}{ Columbidae } \\
\hline Columba picazuro (Temminck, 1813) & Pomba-asa-branca & & A & G \\
\hline Columbina minuta (Linnaeus, 1766) & Rolinha-de-asa-canela & & $\mathrm{E}$ & G \\
\hline Columbina talpacoti (Temminck, 1810) & Rolinha-caldo-de-feijão & & A & G \\
\hline Scardafella squammata (Lesson, 1831) & Fogo-apagou & & $\mathrm{C}$ & G \\
\hline Leptotila verreauxi (Bonaparte, 1855) & Juriti-pupu & & C & G \\
\hline Leptotila rufaxilla (Richard \& Bernard, 1792) & Juriti-gemedeira & & $\mathrm{O}$ & G \\
\hline \multicolumn{5}{|l|}{ Psittacidae } \\
\hline Aratinga leucophthalmus (Statius Muller, 1776) & Maritaca & & $\mathrm{E}$ & $\mathrm{F}$ \\
\hline Aratinga aurea (Gmelin, 1788) & Periquito-cabeça-de-coco & & $A$ & $\mathrm{~F}$ \\
\hline Forpus xanthopterygius (Linnaeus, 1758) & Tuim-de-asa-azul & & A & $\mathrm{F}$ \\
\hline Brotogeris chiriri (Vieillot, 1818) & Periquito-de-asa-amarela & & $\mathrm{C}$ & $\mathrm{F}$ \\
\hline Pionus maximiliani (Kuhl, 1820) & Maitaca-verde & & C & $\mathrm{F}$ \\
\hline \multicolumn{5}{|l|}{ Cuculidae } \\
\hline Coccyzus melacoryphus Vieillot, 1817 & Papa-lagarta & & $\mathrm{O}$ & 1 \\
\hline Piaya cayana (Linnaeus, 1766) & Alma-de-gato & & $\mathrm{C}$ & 1 \\
\hline Crotophaga ani Linnaeus, 1758 & Anu-preto & & $\mathrm{C}$ & I \\
\hline Guira guira (Gmelin, 1788) & Anu-branco & & $\mathrm{C}$ & 1 \\
\hline Tapera naevia (Linnaeus, 1766) & Saci & & $\mathrm{C}$ & 1 \\
\hline \multicolumn{5}{|l|}{ Tytonidae } \\
\hline Tyto alba (Scopoli, 1769) & Suindara & & $\mathrm{O}$ & C \\
\hline \multicolumn{5}{|l|}{ Strigidae } \\
\hline Otus choliba Vieillot, 1817 & Corujinha-do-mato & & $\mathrm{E}$ & $\mathrm{O}$ \\
\hline Bubo virginianus (Gmelin, 1788) & Jacurutu & & $\mathrm{E}$ & $\mathrm{C}$ \\
\hline Glaucidium brasilianum (Gmelin, 1788) & Caburé-ferrugem & & $\mathrm{E}$ & $\mathrm{O}$ \\
\hline \multicolumn{5}{|l|}{ Nyctibiidae } \\
\hline Nyctibius griseus (Gmelin, 1789) & Mãe-da-lua & & $\mathrm{O}$ & 1 \\
\hline \multicolumn{5}{|l|}{ Caprimulgidae } \\
\hline Chordeiles pusillus Gould, 1861 & Bacurau & & $\mathrm{R}$ & 1 \\
\hline Nyctidromus albicollis (Gmelin, 1789) & Curiango & & $\mathrm{C}$ & 1 \\
\hline Caprimulgus rufus Boddaert, 1783 & João-corta-pau & & $\mathrm{C}$ & 1 \\
\hline Caprimulgus longirostris Bonaparte, 1825 & Bacurau-da-telha & & $\mathrm{O}$ & 1 \\
\hline Caprimulgus maculicaudus (Lawrence, 1862) & Bacurau-rabo-maculado & & $\mathrm{E}$ & I \\
\hline Caprimulgus parvulus Gould, 1837 & Bacurau-pequeno & & C & 1 \\
\hline Hydropsalis torquata (Gmelin, 1789) & Curiango-tesoura & & $\mathrm{C}$ & 1 \\
\hline \multicolumn{5}{|l|}{ Apodidae } \\
\hline Streptoprocne zonaris (Shaw, 1796) & Andorinhão-de-coleira & & $\mathrm{E}$ & 1 \\
\hline Cypseloides senex (Temminck, 1826) & Andorinhão-velho-da-cascata & & $\mathrm{E}$ & 1 \\
\hline Chaetura meridionalis Hellmayr, 1907 & Andorinhão-do-temporal & & $\mathrm{E}$ & 1 \\
\hline \multicolumn{5}{|l|}{ Trochilidae } \\
\hline Phaethornis pretrei (Lesson \& Delattre, 1839) * & Rabo-branco-sobre-amarelo & & $\mathrm{C}$ & $\mathrm{N}$ \\
\hline Eupetomena macroura (Gmelin, 1788) & Beija-flor-rabo-tesoura & & C & $\mathrm{N}$ \\
\hline Colibri serrirostris (Vieillot, 1816) * & Beija-flor-de-canto & & $\mathrm{C}$ & $\mathrm{N}$ \\
\hline Anthracothorax nigricollis (Vieillott, 1817) & Beija-flor-de-veste-preta & & $\mathrm{R}$ & $\mathrm{N}$ \\
\hline
\end{tabular}

Revista Brasileira de Zoologia 22 (2): 326-338, junho 2005 
Anexo I. Continuação.

\begin{tabular}{|c|c|c|c|c|}
\hline Famílias e Espécies & Nome Popular & $\begin{array}{c}\text { Conservação e } \\
\text { Endemismo }\end{array}$ & $\begin{array}{l}\text { Abundância } \\
\text { relativa }\end{array}$ & $\begin{array}{l}\text { Guilda } \\
\text { alimentar }\end{array}$ \\
\hline Chlorostilbon aureoventris (Orbigny \& Lafresnave, 1838) & Besourinho-de-bico-vermelho & & $\mathrm{E}$ & $\mathrm{N}$ \\
\hline Thalurania furcata (Gmelin, 1788) & Beija-flor-de-barriga-violeta & & $\mathrm{C}$ & $\mathrm{N}$ \\
\hline Thalurania glaucopis (Gmelin, 1788) & Tesoura-de-fronte-violeta & EMA & $\mathrm{C}$ & $\mathrm{N}$ \\
\hline Amazilia lactea (Lesson, 1829) & Beija-flor-de-peito-azul & & $\mathrm{R}$ & $\mathrm{N}$ \\
\hline Augastes scutatus (Temminck, 1824) * & Beija-flor-de-gravatinha-verde & ENC & $\mathrm{O}$ & $\mathrm{N}$ \\
\hline Heliactin bilophus (Temminck, 1820) & Chifre-de-ouro & & $\mathrm{E}$ & $\mathrm{N}$ \\
\hline Heliomaster squamosus (Temminck, 1823) & Bico-reto-de-banda-branca & & $\mathrm{O}$ & $\mathrm{N}$ \\
\hline Calliphlox amethystina (Boddaert, 1783) & Estrelinha-ametista & & $\mathrm{E}$ & $\mathrm{N}$ \\
\hline \multicolumn{5}{|l|}{ Alcedinidae } \\
\hline Ceryle torquata (Linnaeus, 1766) & Martim-pescador-grande & & $\mathrm{C}$ & $P$ \\
\hline Chloroceryle amazona (Latham, 1790) & Martim-pescador-verde & & C & $P$ \\
\hline Chloroceryle americana (Gmelin, 1788) & Martim-pescador-pequeno & & $\mathrm{O}$ & $P$ \\
\hline \multicolumn{5}{|l|}{ Galbulidae } \\
\hline Galbula ruficauda Cuvier, 1816 & Ariramba-de-cauda-ruiva & & A & 1 \\
\hline \multicolumn{5}{|l|}{ Bucconidae } \\
\hline Nystalus chacuru Vieillot, 1816 * & João-bobo & & $\mathrm{E}$ & I \\
\hline Malacoptila striata (Spix, 1824) & João-barbudo & EMA & $\mathrm{O}$ & I \\
\hline \multicolumn{5}{|l|}{ Rhamphastidae } \\
\hline Ramphastos toco Statius Muller, 1776 & Tucanuçu & & $\mathrm{E}$ & $\mathrm{F} / \mathrm{C}$ \\
\hline \multicolumn{5}{|l|}{ Picidae } \\
\hline Picumnus cirratus Temminck, 1825 & Pica-pau-anão & & A & I \\
\hline Colaptes campestris (Vieillot, 1818) & Pica-pau-do-campo & & A & I \\
\hline Colaptes melanochloros (Gmelin, 1788) & Pica-pau-verde-barrado & & C & I \\
\hline Dryocopus lineatus (Linnaeus, 1766) & Pica-pau-de-banda-branca & & $\mathrm{R}$ & I \\
\hline Melanerpes candidus (Otto, 1796) & Birro & & $\mathrm{E}$ & I \\
\hline Veniliornis passerinus (Linneaus, 1766) & Pica-pauzinho-anão & & $\mathrm{O}$ & I \\
\hline Campephilus melanoleucus (Gmelin, 1788) & Pica-pau-de-topete-vermelho & & $\mathrm{O}$ & I \\
\hline \multicolumn{5}{|l|}{ Formicariidae } \\
\hline Taraba major (Vieillot, 1816) & Choró-boi & & $\mathrm{C}$ & I \\
\hline Thamnophilus pelzeni Hellmayr, 1924 & Choca-bate-cabo-do-sul & & $\mathrm{R}$ & I \\
\hline Thamnophilus caerulescens Vieillot, $1816^{*}$ & Choca-da-mata & & $\mathrm{C}$ & I \\
\hline Dysithamnus mentalis (Temminck, 1823) & Choquinha-lisa & & $\mathrm{R}$ & I \\
\hline Herpsilochmus atricapillus Pelzeln 1868 * & Chororozinho-de-chapéu-preto & & $\mathrm{C}$ & I \\
\hline \multicolumn{5}{|l|}{ Furnariidae } \\
\hline Furnarius rufus (Gmelin, 1788) & João-de-barro & & A & 1 \\
\hline Furnarius figulus (Lichtenstein, 1823) & Casaca-de-couro-da-lama & & $\mathrm{C}$ & I \\
\hline Synallaxis spixi Sclater, 1856 * & João-tenenem & & C & 1 \\
\hline Synallaxis ruficapilla Vieillot, 1819 & Pichororé & EMA & $\mathrm{E}$ & 1 \\
\hline Synallaxis frontalis Pelzeln, 1859 & Petrim & & $\mathrm{E}$ & I \\
\hline Synallaxis albescens Temminck, 1823 & Uipí & & $\mathrm{E}$ & I \\
\hline Certhiaxis cinnamomea (Gmelin, 1788) & Curutié & & $\mathrm{E}$ & I \\
\hline Phacellodomus rufifrons (Wied-NeuWied, 1821) * & João-graveto & & A & I \\
\hline Phacellodomus ruber (Vieillot, 1819) & Graveteiro & & $\mathrm{O}$ & 1 \\
\hline
\end{tabular}


Anexo I. Continuação.

\begin{tabular}{|c|c|c|c|c|}
\hline Famílias e espécies & Nome popular & $\begin{array}{l}\text { Conservação } \\
\text { e endemismo }\end{array}$ & $\begin{array}{c}\text { Abundância } \\
\text { relativa }\end{array}$ & $\begin{array}{l}\text { Guilda } \\
\text { alimentar }\end{array}$ \\
\hline Anumbius annumbi (Vieillot, 1819) & Cochicho & & A & 1 \\
\hline Hylocryptus rectirostris (Wied-NeuWied, 1821) & Fura-barreira & ENC & $E$ & 1 \\
\hline Lochmias nematura (Lichtenstein, 1823) & João-porca & & $E$ & I \\
\hline \multicolumn{5}{|l|}{ Dendrocolaptidae } \\
\hline Lepidocolaptes angustirostris (Vieillot, 1819) & Arapaçu-do-cerrado & & $E$ & 1 \\
\hline \multicolumn{5}{|l|}{ Tyrannidae } \\
\hline Phyllomyias fasciatus (Thunberg, 1822) & Piolhinho & & $\mathrm{O}$ & I \\
\hline Camptostoma obsoletum (Temminck, 1824) & Risadinha & & $\mathrm{R}$ & 1 \\
\hline Phaeomyias murina (Spixi, 1825) & Bagageiro & & A & 1 \\
\hline Suiriri suiriri (Vieillot 1818) * & Suiriri-cinzento & & $\mathrm{R}$ & 1 \\
\hline Myiopagis viridicata (Vieillot, 1817) & Guaracava-de-olheiras & & $\mathrm{R}$ & 1 \\
\hline Elaenia flavogaster (Thunberg, 1822) * & Guaracava-de-barriga-amarela & & A & $\mathrm{I} / \mathrm{F}$ \\
\hline Elaenia spectabilis Pelzeln, 1868 & Guaracava-grande & & $\mathrm{R}$ & $\mathrm{I} / \mathrm{F}$ \\
\hline Elaenia parvirostris Pelzeln, 1868 & Guaracava-de-bico-pequeno & & $\mathrm{R}$ & $\mathrm{I} / \mathrm{F}$ \\
\hline Elaenia mesoleuca (Deppe, 1830) & Tuque & & $\mathrm{R}$ & $\mathrm{I} / \mathrm{F}$ \\
\hline Elaenia cristata Pelzeln, 1868 & Guaracava-de-topete & & C & I/F \\
\hline Elaenia obscura (Orbigny \& Lafresnaye, 1837) * & Tucão & & $\mathrm{C}$ & I/F \\
\hline Elaenia albiceps (Orbigny \& Lafresnaye, 1837) & Guaracava-de-topete-branco & & $\mathrm{O}$ & I/F \\
\hline Elaenia chiriquensis Lawrence, 1865 & Chibum & & $\mathrm{R}$ & $\mathrm{I} / \mathrm{F}$ \\
\hline Leptopogon amaurocephalus Tschudi, 1864 & Cabeçudo & & $\mathrm{E}$ & I \\
\hline Hemitriccus margaritaceiventer (Orbigny \& Lafresnaye, 1837) & Sebinho-olho-de-ouro & & $\mathrm{C}$ & I \\
\hline Todirostrum cinereum (Linnaeus, 1766) & Ferreirinho-relógio & & $\mathrm{O}$ & I \\
\hline Tolmomyias sulphurescens (Spixi, 1825) & Bico-chato-de-orelha-preta & & C & 1 \\
\hline Myiophobus fasciatus (Statius Muller, 1776) & Felipe-de-peito-riscado & & $E$ & 1 \\
\hline Lathrotriccus euleri (Cabanis, 1868) & Enferrujado & & $\mathrm{E}$ & I \\
\hline Cnemotriccus fuscatus (Wied-Neuwied, 1868) & Guaracavuçu & & $\mathrm{E}$ & 1 \\
\hline Xolmis cinerea (Vieillot, 1816) & Maria-branca & & C & 1 \\
\hline Xolmis velata (Lichtenstein, 1823) * & Pombinha-das-almas & & $E$ & 1 \\
\hline Knipolegus lophotes Boie, 1828 & Maria-preta-de-penacho & & $\mathrm{E}$ & I \\
\hline Knipolegus nigerrimus (Vieillot, 1818)* & Maria-p.-de-garganta-vermelha & & E & 1 \\
\hline Fluvicola nengeta (Linnaeus, 1766) & Lavadeira-mascarada & & C & 1 \\
\hline Arundinicola leucocephala (Linnaeus, 1764) & Lavadeira-de-cabeça-branca & & E & 1 \\
\hline Gubernetes yetapa (Vieillot, 1818) & Tesoura-do-brejo & & $\mathrm{C}$ & 1 \\
\hline Satrapa icterophrys (Vieillot, 1818) & Suiriri-pequeno & & $\mathrm{C}$ & 1 \\
\hline Hirundinea ferruginea (Gmelin, 1788) & Gibão-de-couro & & C & 1 \\
\hline Machetornis rixosus (Vieillot, 1819) & Cavaleiro & & $E$ & 1 \\
\hline Casiornis rufa (Vieillot, 1816) & Caneleiro & & $E$ & I \\
\hline Myiarchus ferox (Gmelin, 1789) * & Maria-cavaleira & & $\mathrm{C}$ & 1 \\
\hline Myiarchus tyrannulus (Statius Muller 1776) & Maria-cav.-de-asa-ferrugem & & $\mathrm{E}$ & 1 \\
\hline Myiarchus swainsoni Cabanis e Heine 1859 & Irrê & & $\mathrm{E}$ & 1 \\
\hline Pitangus sulphuratus (Linnaeus 1766) & Bem-te-vi & & A & $\mathrm{O}$ \\
\hline Megarynchus pitangua (Linnaeus, 1766) & Bem-te-vi-de-bico-chato & & $\mathrm{C}$ & 1 \\
\hline Myiozetetes similis (Spix, 1825) & Bem-te-vizinho-coroa-vermelha & & $\mathrm{C}$ & $\mathrm{I} / \mathrm{F}$ \\
\hline
\end{tabular}


Anexo I. Continuação.

\begin{tabular}{|c|c|c|c|c|}
\hline Famílias e espécies & Nome popular & $\begin{array}{c}\text { Conservação e } \\
\text { endemismo }\end{array}$ & $\begin{array}{l}\text { Abundância } \\
\text { relativa }\end{array}$ & $\begin{array}{l}\text { Guilda } \\
\text { alimentar }\end{array}$ \\
\hline Myiodynastes maculatus (Statius Muller, 1776) & Bem-te-vi-rajado & & $\mathrm{C}$ & $\mathrm{I} / \mathrm{F}$ \\
\hline Empidonomus varius (Vieillot, 1818) & Peitica & & $\mathrm{R}$ & I \\
\hline $\begin{array}{l}\text { Griseotyrannus aurantioatrocristatus (Orbigny \& } \\
\text { Lafresnaye, 1837) }\end{array}$ & Peitica-de-chapéu-preto & & $\mathrm{R}$ & I \\
\hline Tyrannus savana Vieillot, 1808 & Tesourinha & & $\mathrm{C}$ & I \\
\hline Tyrannus melancholicus Vieillot, 1819 & Suiriri & & $\mathrm{C}$ & I \\
\hline Tyrannus albogularis Burmeister, 1856 & Suiriri-de-garganta-branca & & $\mathrm{R}$ & I \\
\hline Pachyramphus viridis (Vieillot, 1816) & Caneleirinho-verde & & $\mathrm{R}$ & I \\
\hline Pachyramphus polychopterus (Vieillot, 1819) & Caneleiro-preto & & $\mathrm{E}$ & 1 \\
\hline \multicolumn{5}{|l|}{ Pipridae } \\
\hline Antilophia galeata (Lichtenstein, 1832) & Soldadinho & ENC & $\mathrm{C}$ & $F / I$ \\
\hline \multicolumn{5}{|l|}{ Hirundinidae } \\
\hline Tachycineta leucorrhoa (Vieillot, 1817) & Andorinha-de-sobre-branco & & $\mathrm{E}$ & 1 \\
\hline Progne tapera Boie, 1826 & Andorinha-do-campo & & $\mathrm{E}$ & I \\
\hline Notiochelidon cyanoleuca (Vieillot, 1817) & Andorinha-pequena-de-casa & & $\mathrm{C}$ & I \\
\hline Alopochelidon fucata (Temminck, 1822) & Andorinha-morena & & $\mathrm{E}$ & 1 \\
\hline Stelgidopteryx ruficollis (Vieillot, 1817) & Andorinha-serrador & & $\mathrm{C}$ & 1 \\
\hline \multicolumn{5}{|l|}{ Corvidae } \\
\hline Cyanocorax cristatellus (Temminck, 1823) & Gralha-do-cerrado & ENC & $\mathrm{C}$ & $\mathrm{O}$ \\
\hline \multicolumn{5}{|l|}{ Troglodytidae } \\
\hline Troglodytes musculus Naumann, 1823 & Corruíra & & $\mathrm{C}$ & I \\
\hline \multicolumn{5}{|l|}{ Muscicapidae } \\
\hline Turdus rufiventris (Vieillot, 1818) & Sabiá-laranjeira & & $\mathrm{E}$ & $\mathrm{O}$ \\
\hline Turdus leucomelas (Vieillot, 1818) & Sabiá-barranqueiro & & $\mathrm{C}$ & $\mathrm{O}$ \\
\hline Turdus amaurochalinus (Cabanis, 1850) & Sabiá-poca & & $\mathrm{C}$ & $\mathrm{O}$ \\
\hline \multicolumn{5}{|l|}{ Mimidae } \\
\hline Mimus saturninus (Lichtnstein, 1823) & Arrebita-rabo & & $\mathrm{C}$ & $\mathrm{O}$ \\
\hline \multicolumn{5}{|l|}{ Motacillidae } \\
\hline Anthus lutescens (Pucheran, 1855) & Caminheiro-zumbidor & & $\mathrm{O}$ & I \\
\hline \multicolumn{5}{|l|}{ Vireonidae } \\
\hline Cyclarhis gujanensis (Gmelin, 1789) & Pitiguari & & $\mathrm{C}$ & 1 \\
\hline Hylophilus poicilotis (Temnick, 1822) & Verdinho-coroado & & $\mathrm{O}$ & I \\
\hline \multicolumn{5}{|l|}{ Emberizidae } \\
\hline Parula pitiayumi (Vieillot, 1817) & Mariquita & & $\mathrm{O}$ & I \\
\hline Geothlypis aequinoctialis (Gmelin, 1789) & Pia-cobra & & C & I \\
\hline Basileuterus flaveolus (Baird, SF, 1865) & Pula-pula-amarelo & & A & I \\
\hline Basileuterus culicivorus culicivorus (Deppe, 1830) & Pula-pula-coroado & & $\mathrm{R}$ & I \\
\hline Basileuterus culicivorus hypoleucus Bonaparte, 1850 & Pula-pula-pichito & & $\mathrm{R}$ & I \\
\hline Coereba flaveola (Linnaeus, 1758) & Cambacica & & A & $\mathrm{N}$ \\
\hline Schistochlamys ruficapillus (Vieillot, 1817) * & Bico-de-veludo & & $\mathrm{E}$ & $\mathrm{F} / \mathrm{l}$ \\
\hline Neothraupis fasciata (Lichtenstein, 1823) & Cigarra-do-campo & & $\mathrm{E}$ & $F / I$ \\
\hline Cypsnagra hirundinacea (Lesson, 1831) & Bandoleta & NT & $\mathrm{O}$ & $F / I$ \\
\hline Thlypopsis sordida (Orbigny \& Lafresnave, 1837) & Canário-sapé & & $\mathrm{O}$ & $F / I$ \\
\hline
\end{tabular}


Anexo I. Continuação.

\begin{tabular}{|c|c|c|c|c|}
\hline Famílias e espécies & Nome popular & $\begin{array}{l}\text { Conservação e } \\
\text { endemismo }\end{array}$ & $\begin{array}{l}\text { Abundância } \\
\text { relativa }\end{array}$ & $\begin{array}{l}\text { Guilda } \\
\text { alimentar }\end{array}$ \\
\hline Nemosia pileata (Bodaert, 1783) & Saíra-de-chapéu-preto & & $\mathrm{E}$ & $F / I$ \\
\hline Eucometis penicillata (Spix, 1825) & Pipira-da-taoca & & $\mathrm{O}$ & $F / I$ \\
\hline Piranga flava (Vieillot, 1822) & Sanhaço-de-fogo & & $\mathrm{R}$ & $F / I$ \\
\hline Thraupis sayaca (Linneaus, 1766) & Sanhaço-cinza & & $A$ & $F / I$ \\
\hline Thraupis palmarum (Wied-Neuwied, 1821) & Sanhaço-do-coqueiro & & $\mathrm{E}$ & $F / I$ \\
\hline Euphonia chlorotica (Linneaus, 1766) & Fifi-verdadeiro & & C & $F / I$ \\
\hline Euphonia violacea (Linneaus, 1758) & Gaturamo-verdadeiro & & $\mathrm{O}$ & $F / l$ \\
\hline Tangara cayana (Linneaus, 1766) & Sanhaço-cara-suja & & A & $F / I$ \\
\hline Dacnis cayana (Linneaus, 1766) & Saí-azul & & A & $F / I$ \\
\hline Conirostrum speciosum Temnick, 1824 & Figuinha-de-rabo-castanho & & $\mathrm{O}$ & $F / I$ \\
\hline Tersina viridis (Illiger, 1811) & Saí-andorinha & & $E$ & $F / I$ \\
\hline Zonotrichia capensis (Statius Muller, 1776) & Tico-tico & & $\mathrm{C}$ & G \\
\hline Ammodramus humeralis (Bosc, 1792) * & Tico-tico-do-campo & & A & G \\
\hline Sicalis citrina Pelzeln, 1870 * & Canarinho-rasteiro & & $\mathrm{O}$ & G \\
\hline Emberizoides herbicola (Vieillot, 1817) & Canário-do-campo & & $\mathrm{C}$ & G \\
\hline Volatinia jacarina (Linneaus, 1766) & Tiziu & & $\mathrm{C}$ & G \\
\hline Sporophila plumbea (Wied-Neuwied, 1830) & Patativa-verdadeira & & $\mathrm{R}$ & $G$ \\
\hline Sporophila collaris (Boddaert, 1783) & Coleiro-do-brejo & & $\mathrm{R}$ & G \\
\hline Sporophila lineola (Linnaeus, 1758) & Bigodinho & & $\mathrm{E}$ & G \\
\hline Sporophila nigricollis (Vieillot, 1823) & Coleiro-baiano & & A & G \\
\hline Sporophila caerulescens (Vieillot, 1823) & Coleirinho & & $\mathrm{E}$ & G \\
\hline Sporophila leucoptera (Vieillot, 1817) & Chorão & & $\mathrm{O}$ & G \\
\hline Sporophila bouvreuil (Statius Muller, 1776) & Caboclinho-frade & & $\mathrm{O}$ & G \\
\hline Arremon taciturnus (Hermann, 1783) & Tico-tico-do-mato-bico-preto & & $\mathrm{O}$ & I \\
\hline Arremon flavirostris Swainson, 1838 & Tico-tico-de-bico-amarelo & & $\mathrm{R}$ & I \\
\hline Charitospiza eucosma (Oberholser, 1905) & Mineirinho & ENC NT & $\mathrm{O}$ & G \\
\hline Coryphospingus pileatus (Wied-Neuwied, 1821) & Tico-tico-rei-cinza & & $\mathrm{C}$ & G \\
\hline Saltator similis Orbigny \& Lafresnaye, 1837 & Trinca-ferro & & $\mathrm{C}$ & I \\
\hline Saltator atricollis (Vieillot, 1817) * & Batuqueiro & ENC & $\mathrm{E}$ & I \\
\hline Porphyrospiza caerulescens (Wied-Neuwied, 1830) * & Campainha-azul & ENC & $\mathrm{O}$ & G \\
\hline Psarocolius decumanus (Pallas, 1769) & Japu-preto & & A & $\mathrm{O}$ \\
\hline Agelaius ruficapillus Vieillot, 1819 & Garibaldi & & $\mathrm{R}$ & I \\
\hline Leistes superciliaris (Bonaparte, 1851) & Polícia-inglesa & & $\mathrm{E}$ & I \\
\hline Pseudoleistes guirahuro (Vieillot, 1819) * & Chopim-do-brejo & & $\mathrm{C}$ & I \\
\hline Gnorimopsar chopi (Vieillot, 1819) & Pássaro-preto & & $A$ & I \\
\hline Molothrus bonariensis (Gmelin, 1789) & Gaudério & & E & I \\
\hline
\end{tabular}

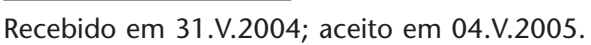

